\title{
EFFECT OF TORSION IN A HELICAL PIPE FLOW
}

\author{
M. VASUDEVAIAH and R. PATTURAJ \\ Department of Mathematics, College of Engineering \\ Anna University, Madras - 600 025, India
}

(Received January 13, 1993)

\begin{abstract}
The problem of fully developed steady viscous incompressible flow in a helical pipe is studied. The predicted analytical expression in the literature for the flow rate is improved. The present result shows a reduction in the flow rate with increasing torsion, for a given curvature. Qualitatively this effect of torsion is seen to cause equivelocity contours in the normal section of the pipe, to undergo shear.
\end{abstract}

KEY WORDS AND PHRASES. Helical pipe, curvature, torsion, flow rate. 1991 AMS SUBJECT CLASSIFICATION CODES.

\section{INTRODUCTION.}

Fluid transportation in helical pipes and spiral coils occurs in many industrial operations involving heat exchangers, chemical reactors etc., and is particularly useful in viscometry or convective heat transfer. Both the earlier experimental investigations of Eustice [1] and the theoretical works of Dean ([2], [3]) have shown a remarkable feature that in a toroidal pipe flow, a fluid particle undergoes a skewed helical motion. Expression for the volumetric flow rate, showing the effect of curvature was later obtained by Topakoglu [4].

Besides curvature $(\kappa)$, torsion $(\tau)$ is another significant parameter which can control the flow in a helical pipe. Assuming that $\kappa a=\varepsilon \ll 1, \tau a=\varepsilon \lambda \ll 1, \lambda=0(1)$, where $a$ is the radius of the circular cross-section of the pipe and using a non-orthogonal curvilinear coordinate system, Wang [5] studied the problem and observed that torsion did not affect the flow rate to the $0\left(\varepsilon^{2}\right)$ considered. His observation of secondary flows, showing asymmetrical recirculating cells which tend to coalesce, thereby reflecting the importance of torsion on the flow, was also not correct, as pointed out by Germano [6].

In the present paper, an attempt is therefore made to bring out the said effect of torsion in a helical pipe flow, assuming the pipe to be nearly straight (the twist of the pipe dominating the bend). The analysis is pursued on similar lines to that of Wang, in terms of the parameter $\delta$, characterizing the helical angle.

\section{ANALYSIS.}

Transport of viscous incompressible fluid, caused due to pressure drop along a helical pipe is considered. The Reynolds number Re characterizing the flow is

$$
R e=U a / \nu,
$$

where the velocity scale $U$ is defined in terms of mean pressure gradient $P$ as

$$
U=\left(a^{2} / 4 \mu\right) P .
$$

Germano's orthogonal coordinate system $\left(s^{\prime}, r^{\prime}, \theta+\phi\left(s^{\prime}\right)+\pi / 2\right)$, Fig. 1, is adopted to describe the mathematical formulation of the problem, with $\phi$ being defined as 


$$
\phi\left(s^{\prime}\right)=-\int_{s_{O}}^{s^{\prime}} \tau\left(s^{\prime}\right) d s^{\prime},
$$

where $\tau\left(s^{\prime}\right)$ measures the torsion of the pipe at $P_{1}$. The corresponding governing equations in dimensionless form are given in detail by Germano [6]. The boundary conditions are the usual no-slip conditions on the body.

The tangent of the helical angle $\beta$ at $P_{1}$ of the central generic curve is related to the curvature $\kappa$ and torsion $\tau$ as

$$
\delta=\tan \beta=\frac{\kappa a}{\tau a}=\frac{\varepsilon}{\sigma}
$$

Both $\sigma$ and $\varepsilon$ are assumed to be small $(\sigma, \varepsilon<1)$ but relatively $\sigma$ is larger than $\varepsilon$. Such a configuration naturally restricts $\delta<1$ unlike that of Wang, so that the twisted pipe considered is nearly straight.

The flow field can now be sought in the form

$$
\begin{gathered}
u(r, \alpha)=\sum_{j=0}^{\infty} \delta^{j} u_{j}(r, \alpha) \\
v(r, \alpha)=\sum_{j=1}^{\infty} \delta^{j} v_{j}(r, \alpha) \\
w(r, \alpha)=\sum_{j=1}^{\infty} \delta^{j} w_{j}(r, \alpha) \\
p(s, r, \alpha)=p_{o}(s)+\sum_{j=1}^{\infty} \delta^{j} p_{j}(r, \alpha) \\
\text { where } \alpha=\theta+\phi .
\end{gathered}
$$

The primary flow of $O\left(\delta^{o}\right)$ is the well-known Poiseuille flow, viz.,

$$
\begin{gathered}
u_{o}(r)=1-r^{2} \\
\text { and } p_{o}(s)=-\frac{4}{R e} s .
\end{gathered}
$$

The first order terms of $O(\delta)$ are solvable from

$$
\begin{gathered}
-\sigma u_{o} \frac{\partial u_{1}}{\partial \alpha}+v_{1} \frac{d u_{o}}{d r}=\sigma r \sin \alpha \frac{d p_{o}}{d x}+\sigma \frac{\partial p_{1}}{\partial \alpha} \\
+\frac{1}{R e}\left[\left(\frac{\partial}{\partial r}+\frac{1}{r}\right)\left(\frac{\partial u_{1}}{\partial r}+\sigma \frac{\partial v_{1}}{\partial \alpha}+\sigma u_{o} \sin \alpha\right)\right. \\
\left.\left.+\frac{1}{r} \frac{\partial}{\partial \alpha}\left(\frac{1}{r} \frac{\partial u_{1}}{\partial \alpha}+\sigma \frac{\partial w_{1}}{\partial \alpha}+\sigma u_{o} \cos \alpha\right)\right)\right] \\
\sigma u_{o} \frac{\partial v_{1}}{\partial \alpha}+\sigma u_{o}^{2} \sin \alpha=\frac{\partial p_{1}}{\partial r} \\
+\frac{1}{R e}\left[\frac{1}{r} \frac{\partial}{\partial \alpha}\left(\frac{\partial w_{1}}{\partial r}+\frac{w_{1}}{r}-\frac{1}{r} \frac{\partial v_{1}}{\partial \alpha}\right)\right. \\
\left.-\sigma \frac{\partial}{\partial \alpha}\left(\frac{\partial u_{1}}{\partial r}+\sigma \frac{\partial v_{1}}{\partial \alpha}+\sigma u_{o} \sin \alpha\right)\right]
\end{gathered}
$$




$$
\begin{aligned}
\sigma u_{o} \frac{\partial w_{1}}{\partial \alpha}+ & \sigma u_{o}^{2} \cos \alpha=\frac{1}{r} \frac{\partial p_{1}}{\partial \alpha} \\
& -\frac{1}{R e}\left[\frac{\partial}{\partial r}\left(\frac{\partial w_{1}}{\partial r}+\frac{w_{1}}{r}-\frac{1}{r} \frac{\partial v_{1}}{\partial \alpha}\right)\right. \\
& \left.+\sigma \frac{\partial}{\partial \alpha}\left(\frac{1}{r} \frac{\partial u_{1}}{\partial \alpha}+\sigma \frac{\partial w_{1}}{\partial \alpha}+\sigma u_{o} \cos \alpha\right)\right) \\
& -\sigma \frac{\partial u_{1}}{\partial \alpha}+\frac{\partial v_{1}}{\partial r}+\frac{v_{1}}{r}+\frac{1}{r} \frac{\partial w_{1}}{\partial \alpha}=0
\end{aligned}
$$

The corresponding solutions obtained exactly up to $O\left(\sigma^{3}\right)$ are given below:

$$
\begin{aligned}
& u_{1}(r, \alpha)=\cos \alpha\left(\sigma^{2} u_{11}\right)+\sin \alpha\left(\sigma u_{12}+\sigma^{3} u_{13}\right) \\
& v_{1}(r, \alpha)=\cos \alpha\left(\sigma^{2} v_{11}\right)+\sin \alpha\left(\sigma v_{12}+\sigma^{3} v_{13}\right) \\
& w_{1}(r, \alpha)=\cos \alpha\left(\sigma w_{11}+\sigma^{3} w_{12}\right)+\sin \alpha\left(\sigma^{2} w_{13}\right) \\
& p_{1}(r, \alpha)=\cos \alpha\left(\sigma^{2} p_{11}\right)+\sin \alpha\left(\sigma p_{12}+\sigma^{3} p_{13}\right)
\end{aligned}
$$

where

$$
\begin{aligned}
& u_{11}=\frac{(R e / 6)}{96} r\left(1-r^{2}\right)\left(29+5 r^{2}-3 r^{4}\right) \\
& +\frac{(R e / 6)^{3}}{134400} r\left(1-r^{2}\right)\left(2969-4381 r^{2}+3249 r^{4}-1301 r^{6}+274 r^{8}-20 r^{10}\right) \\
& u_{12}=-\frac{3}{4} r\left(1-r^{2}\right)+\frac{(R e / 6)^{2}}{320} r\left(1-r^{2}\right)\left(19-21 r^{2}+9 r^{4}-r^{6}\right) \\
& u_{13}=\frac{1}{96} r\left(1-r^{2}\right)\left(6-5 r^{2}\right)-\frac{(R e / 6)^{2}}{7680} r\left(1-r^{2}\right)\left(774-658 r^{2}+\right. \\
& \left.+190 r^{4}+25 r^{6}-8 r^{8}\right)-\frac{(R e / 6)^{4}}{120422400} r\left(1-r^{2}\right)\left(697301-1162699 r^{2}+\right. \\
& \left.+1065233 r^{4}-610567 r^{6}+232037 r^{8}-56083 r^{10}+7757 r^{12}-415 r^{14}\right) \\
& v_{11}=\frac{1}{6}\left(1-r^{2}\right)^{2}+\frac{(R e / 6)^{2}}{1920}\left(1-r^{2}\right)^{2}\left(13-15 r^{2}+7 r^{4}-r^{6}\right) \\
& v_{12}=\frac{(R e / 6)}{48}\left(1-r^{2}\right)^{2}\left(4-r^{2}\right) \\
& v_{13}=\frac{(R e / 6)}{3840}\left(1-r^{2}\right)^{2}\left(189+46 r^{2}-17 r^{4}\right) \\
& +\frac{(R e / 6)^{3}}{15052800}\left(1-r^{2}\right)^{2}\left(11264+1647 r^{2}-6990 r^{4}+4463 r^{6}-1234 r^{8}+125 r^{10}\right) \\
& w_{11}=\frac{(R e / 6)}{48}\left(1-r^{2}\right)\left(4-23 r^{2}+7 r^{4}\right) \\
& w_{12}=\frac{(R e / 6)}{3840}\left(1-r^{2}\right)\left(189+353 r^{2}-207 r^{4}+33 r^{6}\right) \\
& +\frac{(R e / 6)^{3}}{15052800}\left(1-r^{2}\right)\left(11264+281149 r^{2}-537151 r^{4}+458039 r^{6}-205911 r^{8}\right. \\
& \left.+48105 r^{10}-4115 r^{12}\right)
\end{aligned}
$$




$$
\begin{aligned}
w_{13} & =\frac{1}{12}\left(1-r^{2}\right)\left(r^{2}-2\right)-\frac{(R e / 6)^{2}}{1920}\left(1-r^{2}\right)\left(13-224 r^{2}+266 r^{4}\right. \\
& \left.-124 r^{6}+17 r^{8}\right) \\
p_{11}= & \frac{r}{6 R \epsilon}\left(3 r^{2}-1\right)+\frac{(R \epsilon / 6)}{2880}\left(101-120 r^{2}+90 r^{4}-30 r^{6}+3 r^{8}\right) \\
p_{12}= & \frac{r}{12}\left(9-6 r^{2}+2 r^{4}\right) \\
p_{13}= & -\frac{r}{1440}\left(281-255 r^{2}+110 r^{4}-25 r^{6}\right)-\frac{(R e / 6)^{2}}{1612800} r\left(2027-5460 r^{2}+\right. \\
& \left.+5740 r^{4}-3500 r^{6}+1260 r^{8}-252 r^{10}+20 r^{12}\right) .
\end{aligned}
$$

It may be noted that Dean's solutions for a toroidal pipe flow form part of the present solution, and are given by terms of order $\sigma$ in (2.12). Among the second order terms of $O\left(\delta^{2}\right)$, only the aperiodic term $u_{20}$ of the main flow is of interest of us. The detailed equations are too lengthy to be given here.

If we agree to compute the flow rate up to $O\left(\delta^{m} \sigma^{n}\right)_{m+n \leq 6}$, the available solutions (2.6), (2.7), (2.12) are sufficient to calculate $u_{20}$ up to $O\left(\sigma^{4}\right)$. The corresponding solution is

where

$$
u_{20}=\sigma^{2} u_{20}^{(1)}+\sigma^{4} u_{20}^{(2)}+O\left(\sigma^{6}\right)
$$

$$
\begin{aligned}
u_{20}^{(1)} & =\left(1-r^{2}\right)\left[\frac{1}{32}\left(-3+11 r^{2}\right)-\frac{(R e / 6)^{2}}{6400}\left(148+43 r^{2}-132 r^{4}+68 r^{6}-7 r^{8}\right)\right. \\
& \left.-\frac{(R e / 6)^{4}}{2867200}\left(1-r^{2}\right)^{2}\left(4119-8923 r^{2}+7214 r^{4}+2910 r^{6}+535 r^{8}-35 r^{10}\right)\right] \\
u_{20}^{(2)} & =\left(1-r^{2}\right)\left[\frac{1}{2304}\left(49-83 r^{2}+58 r^{4}\right)-\frac{(R e / 6)^{2}}{1843200}\left(82519-148421 r^{2}\right.\right. \\
& \left.+106789 r^{4}-25571 r^{6}-5846 r^{8}+1810 r^{10}\right)-\frac{(R e / 6)^{4}}{260112384000}(145186409 \\
& -214038061 r^{2}+282540539 r^{4}-353746861 r^{6}+313442039 r^{8}-175655185 r^{10} \\
& \left.+60068135 r^{12}-11194585 r^{14}+713090 r^{16}\right) \\
& +\frac{(R e / 6)^{6}\left(1-r^{2}\right)^{3}}{38149816320000}\left(3068498717-4237343932 r^{2}+3407539940 r^{4}\right. \\
& -1828254380 r^{6}+675698470 r^{8}-170804372 r^{10}+27992412 r^{12} \\
& \left.\left.-2606580 r^{14}+96525 r^{16}\right)\right]
\end{aligned}
$$

\section{FLOW RATE.}

The volume rate of discharge of the fluid through the circular cross-section of the pipe is given in terms of dimensionless variables as

$$
\frac{q}{U a^{2}}=\int_{o}^{2 \pi} \int_{o}^{1} u r d r d \alpha .
$$

Dropping the periodic terms which do not contribute to the integral, the above expression (3.1) simplifies, to

$$
\frac{q}{2 \pi U a^{2}}=Q=\int_{o}^{1}\left[u_{o}(r)+\delta^{2}\left\{\sigma^{2} u_{20}^{(1)}+\sigma^{4} u_{20}{ }^{(2)}\right\}\right] r d r
$$


The integral (3.2) is evaluated exactly using (2.6), (2.26), (2.27) to give

where

$$
\frac{Q}{Q_{S}}=1-\frac{\delta^{2} \sigma^{2}}{48}\left[\frac{1541}{67200}(R e / 6)^{4}+\frac{11}{10}(R e / 6)^{2}-1\right]+\frac{\delta^{2} \sigma^{4}}{48} f(R e)
$$

$$
f(R e)=\frac{12483167}{9934848000}(R e / 6)^{6}-\frac{1189733}{64512000}(R e / 6)^{4}-\frac{8397}{6720}(R e / 6)^{2}+\frac{31}{48}
$$

and $Q_{S}$ is the corresponding flow rate in a straight tube.

\section{DISCUSSION.}

The analytical expression (3.3) shows the combined effect of $\delta$ and $\sigma$ on the flow rate. If we write the expression in terms of $\epsilon$, the last terms of $O\left(\epsilon^{2} \sigma^{2}\right)$ marked by an asterisk can be looked upon as a simple addition (due to torsion) to Wang's [5] result, when $\lambda=1 / \delta=0(1)$.

We now look at the quantitative effect of torsion compared with that of curvature on the flow rate. Table 1 gives a comparative statement of the existing flow rate $Q_{w}$, Wang [5] and the present improvement $Q$ for a selected value of $\delta=0.2$ (the corresponding helical angle is $\beta=11.31^{\circ}$ ). The numerical values of $Q_{w}$ show that for small $\epsilon$, when $R e<6$ (more precisely 5.67) the flow rate in a curved pipe is larger than that of a straight pipe, as remarked earlier by Wang. Such an observation can be made in the present case also as the value of $R e$ approaches 5 or 6. Comparatively $Q>Q_{w}>1$ when $R e<5$. This inequality is observed true for different $\delta<1$ (the detailed numerical data is not supplied). For $R e>5.67, Q<Q_{w}<1$. However, the range of validity of the expression (3.3) for the flow rate, cannot be indefinitely extended to much larger values of $R e$. This can be seen more clearly from the following discussion.

Table 2 gives the flow rates for a given Reynolds number $R e=17$ and $\epsilon=0.1,0.2$ and 0.5 . The flow rates decrease with increasing $\sigma$ (torsion) for a given curvature. This decrease is more pronounced with increasing values of curvature. Similar computations given in Table 3 show an increase in the flow rates with increasing $\sigma$, for some values of the Reynolds number $(\epsilon=0.1, R e=53.76$ and $\epsilon=0.2, R e=38.01)$. This appears physically inconsistent**. This is because the values of the Reynolds number in these cases have been chosen as $R e=17 / \sqrt{ } \epsilon$, an optimum value suggested by Wang for the validity of his flow rate. This only shows that $R e=17 / \sqrt{ } \epsilon$ cannot be taken as an upper bound in the present case also, since it involves an additional term of $O\left(\epsilon^{2} \sigma^{2}\right)$ which seems to diminish the value of the upper bound.

Since the secondary flow cannot be described by a stream function in the present case, the deviation of stream lines compared to toroidal pipe flow cannot be studied, to interpret the effect of torsion on the flow. Instead, the displacement of equivelocity contours is studied. While the study of stream lines will help us in picturing the flow structure, the equivelocity contours will help us to fix the spatial positions of characteristic particles (particles having the same velocity) which are displaced from their original configurations. This displacement should be therefore due to the inherent property of the torsion causing a rigid body-like twist to the fluid particles. To illustrate this feature, equivelocity profiles in $(r, \alpha)$ plane of Wang (corrected to correlate with physical covariant description) and their respective deviations in the present case are projected in Figure 2, for selected values of $\delta=0.2, \sigma=0.25, R e=6$ and 38. The resultant secondary velocity $\sqrt{v^{2}+w^{2}} \approx \delta \sqrt{v_{1}^{2}+w_{1}^{2}}$ is computed both for its magnitude and direction, using the analytical expression (2.12), with $r$ and $\alpha$ ranging from 0.05 to 0.95 and $O$ to $2 \pi$ respectively. The corresponding equivelocity contours are then sketched. The numerical data is too voluminous to be presented here. Figures $(2 a, b)$ show how equivelocity profiles of Wang get sheared and accelerated. With increase in $R e$, relatively stagnant profiles of Wang tend to reach the wall. The resistance of the wall coupled with increased flow (due to increase of pressure gradient) 
surges the shearing, reversing its orientation. Figures $(2 c, d)$ reflect this phenomena. The said shearing of equivelocity profiles of Wang signifies the torsional effect on the helical pipe flow, which is otherwise reflected in the volume flow rate.

Table 1. Flow rate in a helical pipe
$\delta=\epsilon / 0=0.2$

\begin{tabular}{|c|c|c|c|c|c|c|}
\hline Ne & $\mathbf{Q}_{\mathbf{w}}$ & Q & $\mathbf{Q}_{\mathbf{w}}$ & $\mathbf{Q}$ & $\mathbf{Q}_{\mathbf{w}}$ & $\mathbf{Q}$ \\
\hline 1 & 1.00005054 & 1.00005257 & 1.00020194 & 1.00023377 & 1.00080788 & 1.00131714 \\
\hline 2 & 1.00004566 & 1.00004733 & 1.00018287 & 1.00020921 & 1.00073123 & 1.00115359 \\
\hline 3 & 1.00003767 & 1.00003874 & 1.00015080 & 1.00016809 & 1.00060296 & 1.00087988 \\
\hline 4 & 1.00002635 & 1.00002658 & 1.00010550 & 1.00011003 & 1.00042212 & 1.00049460 \\
\hline 5 & 1.00002168 & 1.00001097 & 1.00004685 & 1.00003481 & 1.00018752 & 0.99999553 \\
\hline 6 & 0.99999362 & 0.99999160 & 0.99997437 & 0.99994200 & 0.99989754 & 0.99938011 \\
\hline 7 & 0.99997187 & 0.99996835 & 0.99988759 & 0.99983102 & 0.99955022 & 0.99864525 \\
\hline 8 & 0.99994648 & 0.99994117 & 0.99978584 & 0.99970108 & 0.99914330 & 0.99778759 \\
\hline 9 & 0.99991715 & 0.99990982 & 0.99966854 & 0.99955165 & 0.99867409 & 0.99680352 \\
\hline 10 & 0.99988371 & 0.99987411 & 0.99953490 & 0.99938178 & 0.99813956 & 0.99568915 \\
\hline 15 & 0.99964738 & 0.99962270 & 0.99858940 & 0.99819475 & 0.99435771 & 0.98804513 \\
\hline 20 & 0.99926805 & 0.99922317 & 0.99707222 & 0.99635392 & 0.98828894 & 0.97679621 \\
\hline 25 & 0.99869746 & 0.99863225 & 0.99478978 & 0.99374646 & 0.97915918 & 0.96246636 \\
\hline 30 & 0.99787331 & 0.99780011 & 0.99149328 & 0.99032211 & 0.96597314 & 0.94723445 \\
\hline
\end{tabular}

Table 2. Effect of torsion on flow rate : $R e=17$

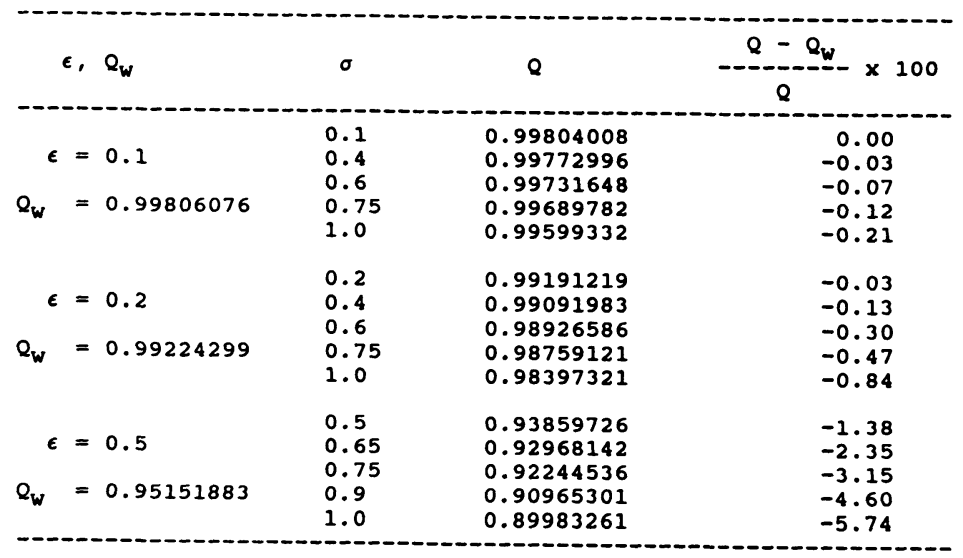


Table 3. Effect of Torsion on rlow Rate: Re $=17 / \mathrm{J}$

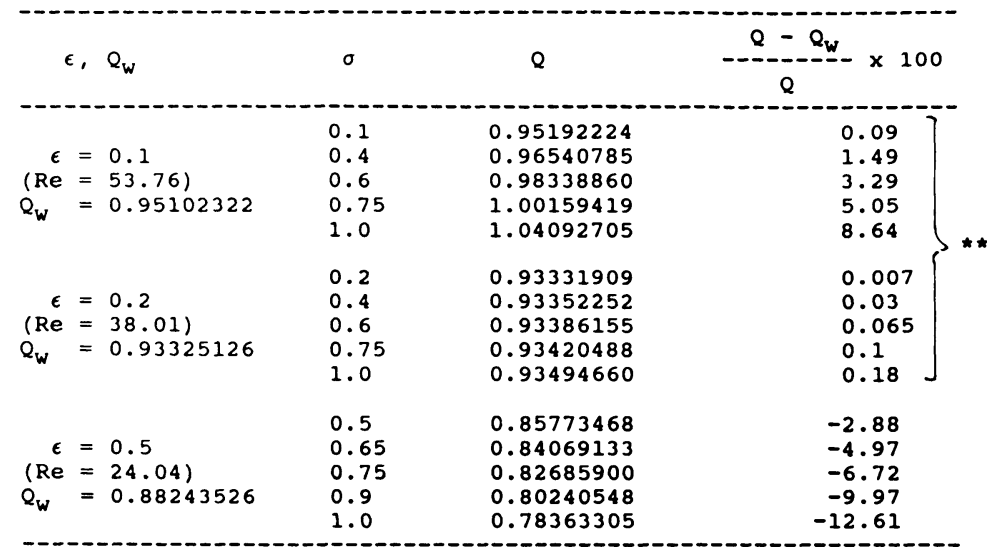

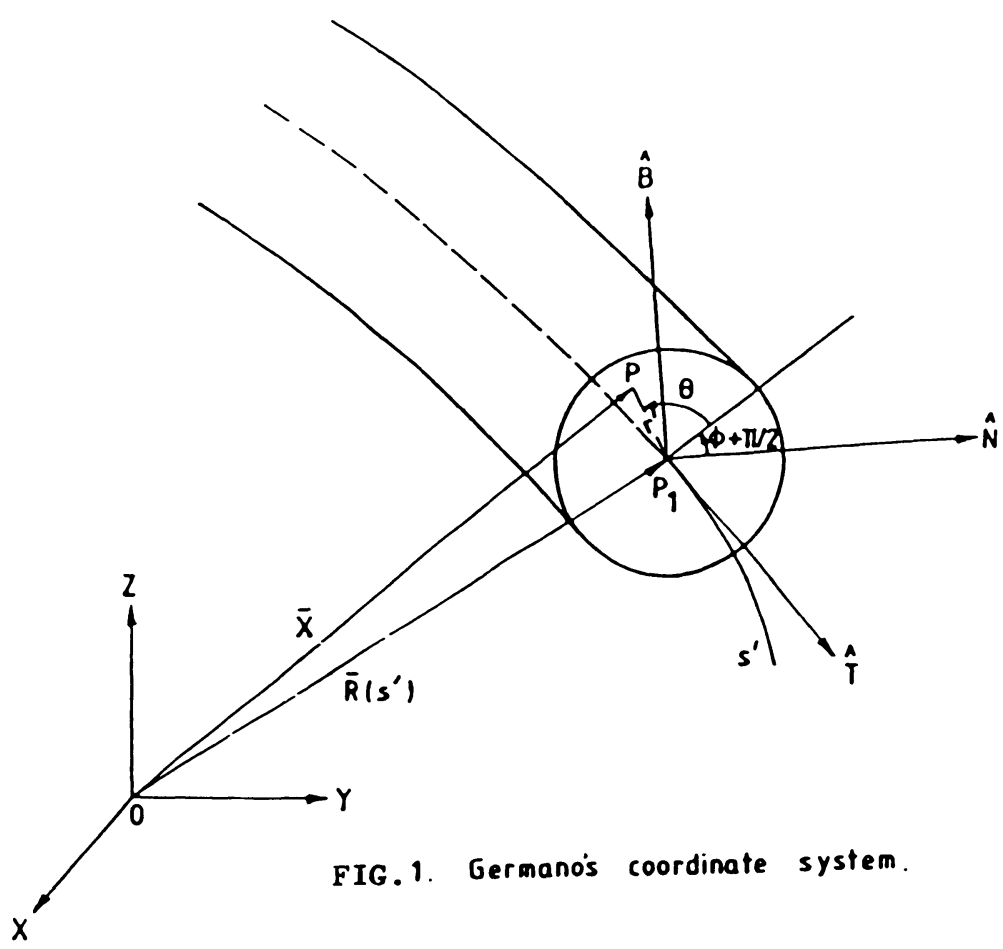




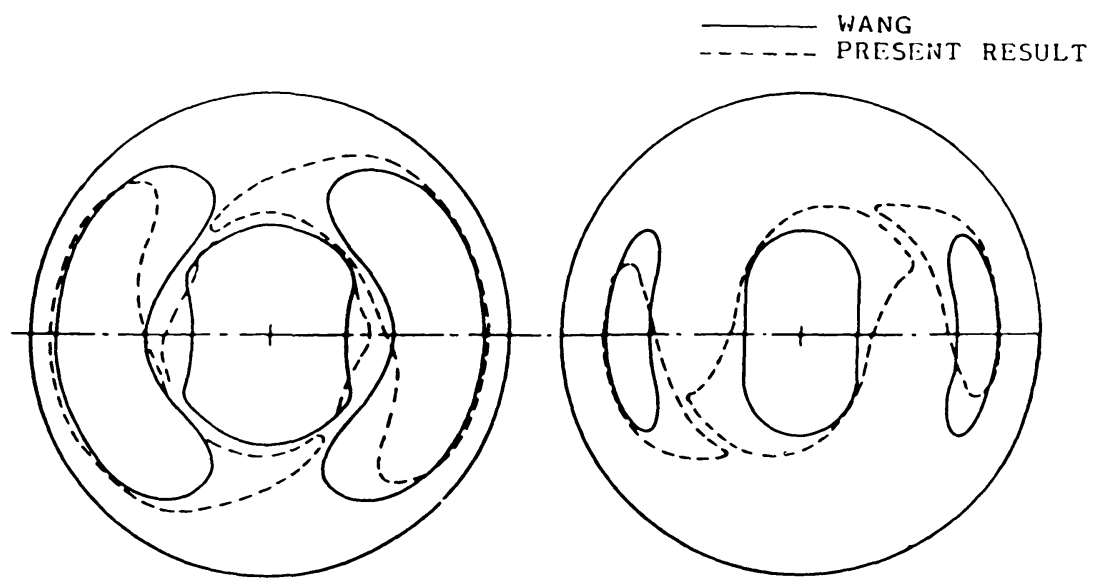

a)

b)

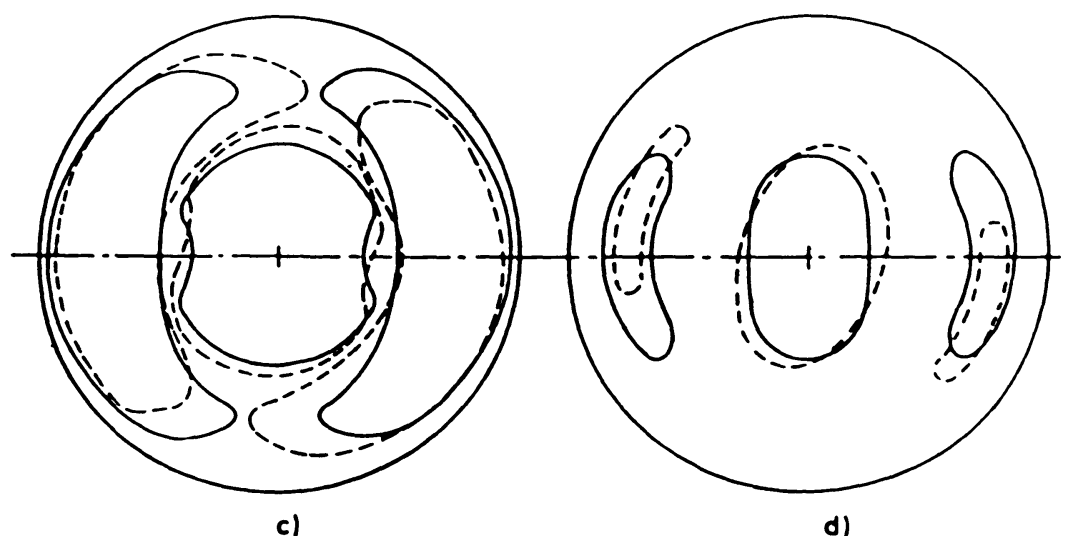

FIG. 2 EQUIVELOCITY CONTOURS IN $(r, a)$ PLANE: $\delta=0.2, \sigma=025$,

Re $=6$ : a) $\sqrt{v^{2}+w^{2}}=0.0015$ b) $\sqrt{v^{2}+w^{2}}=0.0026$

$R_{e}=38$ : c) $\sqrt{v^{2}+w^{2}}=0.007 \quad$.d) $\sqrt{v^{2}+w^{2}}=0.016$

\section{REFERENCES}

1. EUSTICE, J., Flow of water in curved pipes, Proc. Royal. Soc. A84 (1910), 107-119.

2. DEAN, W.R., Note on the motion of fluid in a curved pipe, Phil. Mag. and J. Sci. IV (1927), 208-223.

3. DEAN, W.R., The stream-line motion of fluid in a curved pipe, Phil. Mag. and J. Sci. V (1928), 673-695.

4. TOPAKOGLU, H.C., Steady laminar flows of an incompressible viscous fluid in curved pipes, J. Math. \& Mech. 16 (1967), 1321-1337.

5. WANG, C.Y., On the low-Reynolds-number flow in a helical pipe, J. Fluid Mech. 108 (1981), 185-194.

6. GERMANO, M., On the effect of torsion on a helical pipe flow, J. Flurd Mech. 125 (1982), $1-8$. 


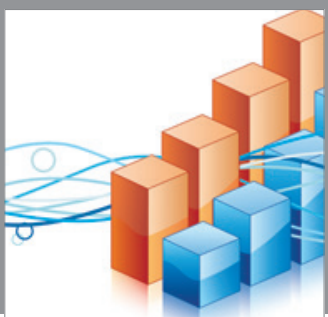

Advances in

Operations Research

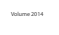

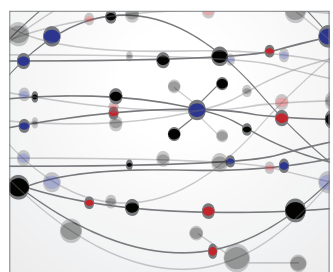

\section{The Scientific} World Journal
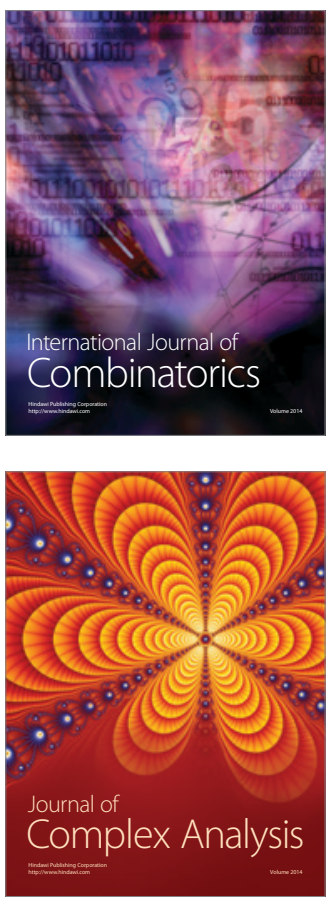

International Journal of

Mathematics and

Mathematical

Sciences
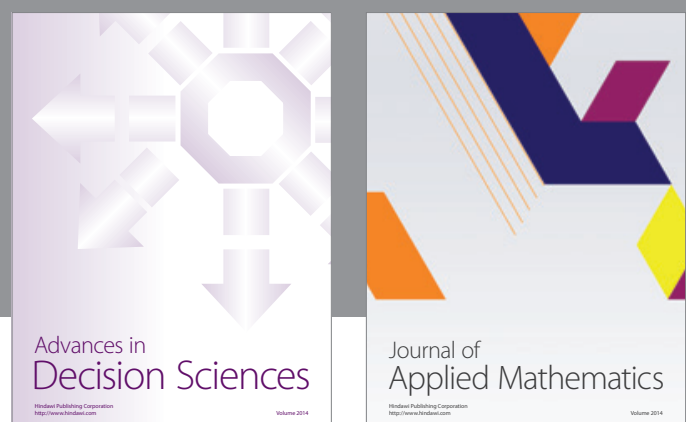

Journal of

Applied Mathematics
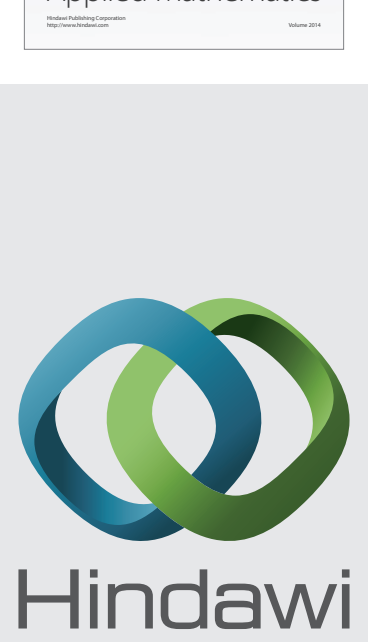

Submit your manuscripts at http://www.hindawi.com
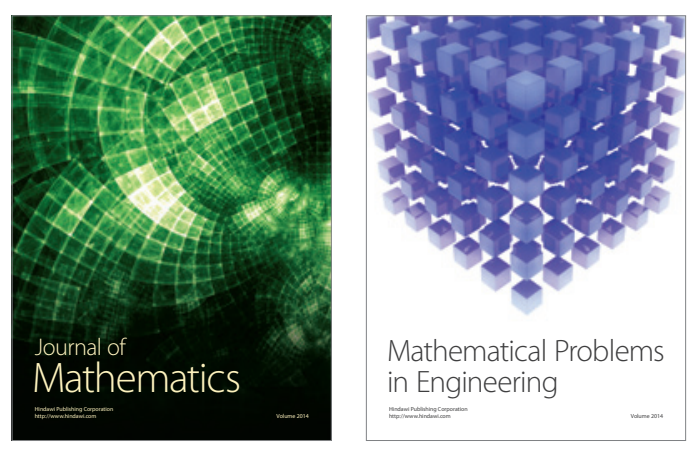

Mathematical Problems in Engineering
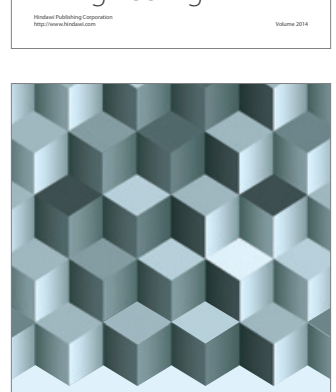

Journal of

Function Spaces
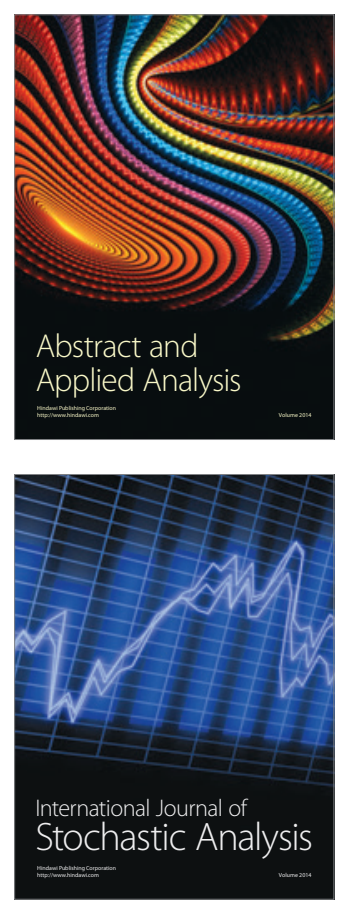

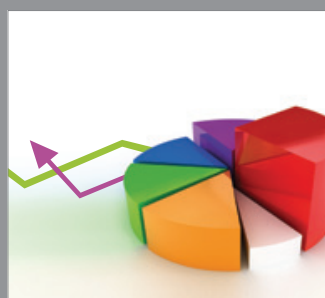

ournal of

Probability and Statistics

Promensencen
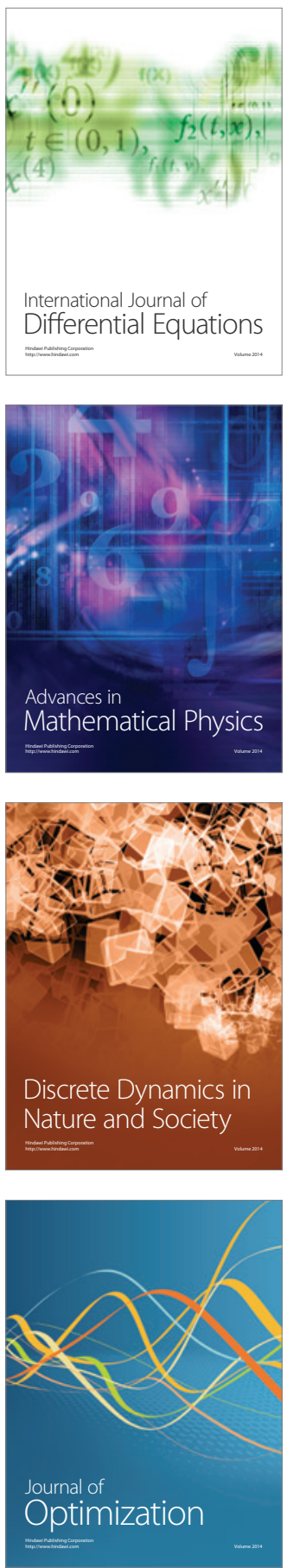